\title{
CERTAIN POTENTIAL FUNCTIONS AND A NEW SOLUTION OF LAPLACE'S EQUATION
}

\author{
By T. J. I'A. Bromwich.
}

[Read June 13th, 1912.-Received September 5th, 1912.]

Ir is a familiar fact that the potential of a uniform circular disc of radius $a$ can be expressed by series of zonal harmonics of the form

$$
\sum A_{n} P_{n}(\mu) / r^{n+1} \text { or } \sum B_{n},^{n} P_{n}(\mu),
$$

according as $r$ is greater or less than $a$. And a similar statement bolds for the potential of a uniform hemisphere (with a slight modification at internal points); the exact formulæ for both cases are given below (pp. 103 and 120).

There is no difficulty in shewing that, if $r>a$, a series of the type $\Sigma A_{n} P_{n}(\mu) / r^{n+1}$ will serve to represent the potential, whatever the law of density in the dise or hemisphere may be, provided that it is symmetrical.*

But when the density is not uniform, the series for points at which $r<a$ become more complicated : in particular, if the density is an odd power of the distance from the centre certain new types of harmonic (here denoted by $\left.X_{n}\right)$ will appear, containing terms of the type $r^{n} P_{n}(\mu) \log (a / r)$. The precise form of $X_{n}$ is investigated in $\$$ II below.

The formulæ for the potentials in $\$$ I, VI and the formulie for $X_{n}$ were obtained four years ago, but were first published in the Abstracts of the Proceedings of the June Meeting of the Society; after these Abstracts had appeared, it was remarked to me by Mr. G. N. Watson that the function $X_{n}$ is connected with the function

$$
Y_{n}=\frac{\partial}{\partial n}\left\{\left(\frac{v}{a}\right)^{n} P_{n}(\mu)\right\},
$$

which is a more obvious solution of Laplace's equation. A verification of this fact will be found in $\S \mathrm{V}$ below ; and some of the potential functions

\footnotetext{
* More generally, a series of this type can be found for any solid of revolution, if $r$ is greater than the greatest radius of the solid.
} 
have been now expressed in terms of ' $Y_{n}$ rather than in terms of $X_{n}$, as this enables these formulæ to be written more compactly.

\section{Potential of a Disc whose Density Varies as $\mathrm{s}^{\mathrm{nt}}$, where $s$ is the Distance from the Centre.}

We take the centre of the disc as origin and its plane as the plane of $x y$. Then the potential is given by the integral

$$
V=\int_{0}^{2 \pi} d \phi \int_{0}^{a} s d s \frac{k s^{m}}{\left.\sqrt{\left(r^{2}\right.}+s^{2}-2 r s \cos \gamma\right)},
$$

where $\cos \gamma=\sin \theta \cos \phi$, and $r, \theta$ are the three dimensional polar coordinates of the point at which $V$ is to be found.

We can expand the square root in a series proceeding according to powers of $s / r$ (or $r / s$, as the case may be), so that

$$
\begin{array}{r}
\frac{1}{\sqrt{\left(r^{2}+s^{2}-2 r s \cos \gamma\right)}}=\frac{1}{r}+\sum_{1}^{\infty} \frac{s^{n}}{r^{n+1}} P_{n}(\cos \gamma), \text { if } s<r, \\
\text { or } \frac{1}{s}+\sum_{1}^{\infty} \frac{r^{n}}{s^{n+1}} P_{n}(\cos \gamma), \text { if } s>r .
\end{array}
$$

Also it is known that

$$
\int_{0}^{2 \pi} P_{n}(\cos \gamma) d \phi=2 \pi P_{n}(0) P_{n}(\cos \theta),
$$

and that

$$
P_{n}(0)=0 \text {, if } n \text { is odd, }
$$

or

$$
P_{i n}(0)=(-1)^{m} \frac{1.3 \ldots(n-1)}{2.4 \ldots n} \text {, if } n \text { is even }=2 m \text {. }
$$

For brevity in the subsequent work we write

$$
C_{i}=\frac{1.3 \ldots(2 n-1)}{2.4 \ldots 2 n},
$$

and then

$$
P_{2 i l}(0)=(-1)^{n} C_{n} \text {. }
$$

On using these facts we get two formulæ for $V$, according as $r$ is greater or less than $a$, which we shall denote by $V_{0}$ and $V_{1}$ respectively; thus, if $r>a$, we have

(1) $V_{0}=2 \pi k \int_{0}^{a} s^{m+1} d s\left\{\frac{1}{r}+\sum_{1}^{\infty}(-1)^{n} C_{n} \frac{s^{2 n}}{r^{2 n+1}} P_{2 n}(\cos \theta)\right\}$

$$
=2 \pi k a^{m+1}\left\{\frac{1}{m+2} \frac{a}{r}+\sum_{1}^{\infty}(-1)^{n} \frac{C_{n}}{m+2 n+2}\left(\frac{a}{r}\right)^{2 n+1} P_{2 n}(\cos \theta)\right\} .
$$

But, if $r<a$, we have to divide the integral into two parts, from 0 to $r$, 
and from $r$ to $a$, and then we find

$$
\begin{aligned}
V_{1}= & 2 \pi k \int_{0}^{r} s^{m+1} d s\left\{\frac{1}{r}+\sum_{1}^{\infty}(-1)^{n} C_{n} \frac{s^{2 n}}{r^{2 n+1}} P_{2 n}(\cos \theta)\right\} \\
& +2 \pi k \int_{r}^{a} s^{n+1} d s\left\{\frac{1}{s}+\sum_{1}^{\infty}(-1)^{n} C_{n} \frac{r^{2 n}}{s^{2 n+1}} P_{2 n}(\cos \theta)\right\} .
\end{aligned}
$$

Thus, assuming that $m$ is not an odd integer (we have tacitly assumed already that $m+2>0$, so that the values excluded now are $-1,+1$, $+3,+5, \ldots)$, we find the result

$$
\begin{aligned}
V_{1}= & 2 \pi k r^{m+1}\left\{\frac{-1}{(m+1)(m+2)}+\sum_{1}^{\infty}(-1)^{n} \frac{C_{n}(4 n+1) P_{2 n}(\cos \theta)}{(2 n+m+2)(2 n-m-1)}\right\} \\
& +2 \pi k a^{m+1}\left\{\frac{1}{m+1}-\sum_{1}^{\infty}(-1)^{n} \frac{C_{n}}{2 n-m-1}\left(\frac{r}{a}\right)^{2 n} P_{2 n}(\cos \theta)\right\}
\end{aligned}
$$

Since $V_{1}$ satisfies Laplace's equation, we are led to conjecture that the first term in $V_{1}$ must be of the form $2 \pi k A r^{m+1} P_{m+1}(\cos \theta)$; but (since $m+1$ is not an even integer) this function has not the property of remaining unchanged when we change the sign of $\cos \theta$. Thus we amend our conjecture by trying to express the term in the form

or

$$
\begin{array}{rll}
2 \pi k A r^{m+1} P_{m+1}(\cos \theta), & \text { when } & \cos \theta>0, \\
2 \pi k A r^{m+1} P_{m+1}(-\cos \theta), & \text { when } & \cos \theta<0 ;
\end{array}
$$

and this function has the further property of remaining finite in both regions, even when $m$ is not an integer.

It is now easy to confirm the conjecture, for if we write

$$
P_{m+1}(|\mu|)=B_{0}+\sum_{1}^{x} B_{n} P_{n}(\mu),
$$

where

$$
\mu=\cos \theta \text {, }
$$

we get

$$
2 B_{0}=\int_{-1}^{+1} P_{m+1}(|\mu|) d \mu=2 \int_{0}^{1} P_{m+1}(\mu) d \mu,
$$

and so

$$
B_{0}=-\frac{1}{(m+1)(\overline{m+2)}}\left[\left(1-\mu^{2}\right)-\frac{d P_{m+1}}{d \mu}\right]_{0}^{1}=-\frac{p}{(m+1)(m+2)}
$$

where

$$
p=-\left[\frac{d P_{m+1}}{d \mu}\right]_{\mu=0}
$$

Also

$$
\begin{aligned}
\frac{2 B_{n}}{2 n+1}=\int_{-1}^{+1} P_{m+1}(|\mu|) P_{n}(\mu) d \mu & =0 \quad \text { (if } n \text { is odd) } \\
& =2 \int_{0}^{1} P_{m+1}(\mu) P_{n}(\mu) d_{\mu} \quad \text { (if } n \text { is even). }
\end{aligned}
$$

Now

$$
\{n(n+1)-(m+1)(m+2)\} \int P_{m+1} P_{n} d \mu=\left(1-\mu^{2}\right)\left(P_{n} \frac{d P_{m+1}}{d \mu}-P_{m+1} \frac{d P_{n}}{d \mu}\right),
$$


by using Legendre's equation for the harmonics. Also, when $n$ is even, $d P_{n} / d \mu$ is zero at $\mu=0:$ and so we find

$$
B_{n}=\frac{(2 n+1) p P_{n}(0)}{(n-m-1)(n+m+2)} \quad \text { (if } n \text { is even). }
$$

Thus

and so we get the result

$$
B_{n_{n}}=\frac{(4 n+1) p(-1)^{n} C_{n}}{(2 n-m-1)(2 n+m+2)}
$$

$$
P_{m+1}(|\mu|)=p\left\{-\frac{1}{(m+1)(m+2)}+\sum_{1}^{\infty} \frac{(-1)^{n} C_{n}(4 n+1) P_{2 n}}{(2 n-m-1)(2 n+m+2)}\right\} .
$$

Thus the potential of the disc, when $r<a$, takes the form

$$
\begin{aligned}
V_{1}= & 2 \pi(k / p) r^{m+1} P_{n+1}(|\mu|) \\
& +2 \pi k a^{n+1}\left\{\frac{1}{m+1}-\sum_{1}^{\infty}(-1)^{n} \frac{C_{n}}{2 n-m-1}\left(\frac{r}{a}\right)^{2 n} P_{2 n}(\cos \theta)\right\},
\end{aligned}
$$

where

$$
p=-\left(\frac{d P_{m+1}}{d \mu}\right)_{0}=-\frac{2}{\sqrt{ } \pi} \cos \left(\frac{1}{2} m \pi\right) \frac{\Gamma\left(\frac{1}{2} m+\frac{3}{2}\right)}{\Gamma\left(\frac{1}{2} m+1\right)} \quad \text { (see p. 104), }
$$

$\mathrm{OI}^{*}$

$$
\frac{1}{p}=-\frac{1}{(m+1)(m+2)}+\sum_{1}^{\infty} \frac{(-1)^{n} C_{n}(4 n+1)}{(2 n+m+2)(2 n-m-1)},
$$

the second formula for $p$ being obtained by equating the series (3) and (4) in the special case $\theta=0$.

The form (3) enables us to see at a glance the continuity of $V$ and $\partial V / \partial r$ at the sphere $r=a$; but (4) is useful also, as indicating at once the fact that $V$ satisfies Laplace's equation.

As an illustration we may refer to the familiar case of a uniform disc, for which $m=0$; here $p=-1$, and so (4) gives

$$
V_{1}=-2 \pi k r \cdot|\mu|+2 \pi k a\left\{1-\sum_{1}^{\infty}(-1)^{n} \frac{C_{n}}{2 n-1}\left(\frac{r}{a}\right)^{2 n} P_{2 n}\right\},
$$

while (1) gives $V_{0}=2 \pi k a\left\{\frac{1}{2} \frac{a}{r}+\sum_{1}^{\infty}(-1)^{n} \frac{C_{n}}{2 n+2}\left(\frac{a}{r}\right)^{2 n+1} P_{2 n}\right\}$.

The continuity at $r=a$ of these formulæ was established by a special investigation (here extended) in the Philosophical Magazine (August, 1901); it will be seen that our present formulæ are in agreement with those found in the ordinary text books, except for the fact that some books seem to suppose that the first term in $V_{1}$ is always $-2 \pi k r \mu$.

As a further verification of (4), it may be noted that the discontinuity in the normal attraction at the surface of the disc leads to the equation

$$
\left(\frac{\partial V_{1}}{\partial z}\right)_{-}\left(\frac{\partial V_{1}}{\partial z}\right)_{+}=4 \pi \sigma
$$


or, in our case,

$$
-\frac{1}{r}\left(\frac{\partial V_{1}}{\partial \mu}\right)_{+}=2 \pi \sigma=2 \pi k r^{m}
$$

which agrees with (4).

We now proceed to discuss the necessary modification of (4), when $m$ is an odd integer; and, on the whole, the simplest method appears to be the application of a limiting process. To carry this out, we shall need to establish first the formula given in (4) for the value of the constant $p$.

In general the relation

$$
\left(1-\mu^{2}\right) \frac{d P_{n}}{d \mu}=n\left(P_{n-1-\mu} P_{n+1}\right)
$$

is easily established for all values of $n$; and so, putting $\mu=0$ and $n=m+1$, we find

$$
-p=(m+1) P_{m}(0) \text {. }
$$

Alsu, when $\mu$ is positive, we have

$$
P_{m}(\mu)=\frac{1}{\pi} \int_{0}^{\pi}\left\{\mu+\omega^{\prime}\left(1-\mu^{2}\right) \cos \phi\right\}^{\prime \prime \prime} d \phi,
$$

where the principal value of the complex power is to be used. Thus, if

we have as the limiting value

$$
\mu \rightarrow 0 \text { and } 0 \leqslant \phi \leqslant \frac{1}{2} \pi,
$$

$$
\left\{\mu+\iota \sqrt{ }\left(1-\mu^{\prime \prime}\right) \cos \phi\right\}^{\prime \prime \prime} \rightarrow e^{3 \mathrm{~s} u \prime \prime} \cos ^{\prime \prime \prime} \phi,
$$

but when $\frac{1}{2} \pi \leqslant \phi \leqslant \pi$, the formula is

$$
\left\{\mu+\cdots /\left(1-\mu^{2}\right) \cos \phi\right\}^{\prime \prime} \rightarrow e^{-\frac{1}{2} m m r} \cos ^{m \prime} \phi_{1}, \text { if } \phi_{1}=\pi-\phi .
$$

Consequently we find

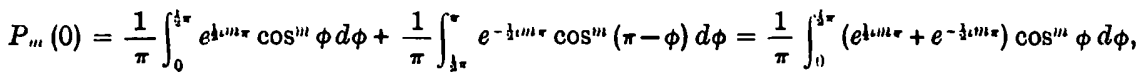

$$
\begin{aligned}
& \text { or } \\
& P_{m}(0)=\frac{2}{\pi} \cos \left(\frac{1}{2} m \pi\right) \int_{0}^{j *} \cos ^{m} \phi d \phi=\frac{1}{\sqrt{ } / \pi} \cdot \cos \left(\frac{1}{2} m \pi\right) \frac{\Gamma\left(\frac{1}{2} m+\frac{1}{2}\right)}{\Gamma\left(\frac{1}{2} m+1\right)} . \\
& -p=(m+1) P_{m}(0)={ }_{\sqrt{\prime}}^{2} \cos \left(\frac{1}{2} m \pi\right) \frac{\Gamma\left(\frac{1}{2} m+\frac{3}{2}\right)}{\Gamma\left(\frac{2}{2} m+1\right)},
\end{aligned}
$$

which is the result already stated in (4) above.

'To deal with the limiting case, it is convenient to write $m+1=2 \nu+t$, and then to make $t$ tend to zero; and a glance at (4) shews that we shall need to evaluate $p$ as far as terms in $t^{2}$. Thus we write to this order

$$
\cos \left(\frac{1}{2} m \pi\right)=\sin \left(\nu \pi+\frac{1}{2} t \pi\right)=(-1)^{\nu} \sin \left(\frac{1}{2} t \pi\right)=(-1)^{\nu}\left(\frac{1}{2} t \pi\right),
$$

and so the quotient of the two Gamma-functions need not be evaluated beyond terms of the first degree in $t$. Thus we can use the approximate formulæ

$$
\begin{array}{ll} 
& \Gamma\left(\frac{1}{2} m+\frac{3}{2}\right)=\Gamma\left(\nu+1+\frac{1}{2} t\right)=\Gamma(\nu+1)\left\{1+\frac{1}{2} t \psi(\nu+1)\right\}, \\
\text { and } & \Gamma\left(\frac{1}{2} m+1\right)=\Gamma\left(\nu+\frac{1}{2}+\frac{1}{2} t\right)=\Gamma\left(\nu+\frac{1}{2}\right)\left\{1+\frac{1}{2} t \psi\left(\nu+\frac{1}{2}\right)\right\}, \\
\text { where } & \psi(x)=\Gamma^{\prime}(x) / \Gamma(x) . \\
\text { Thus } & \frac{\Gamma\left(\frac{1}{2} m+\frac{3}{2}\right)}{\Gamma\left(\frac{1}{2} m+1\right)}=\frac{\Gamma(\nu+1)}{\Gamma\left(\nu+\frac{1}{2}\right)}\left[1+\frac{1}{2} t\left\{\psi(\nu+1)-\psi\left(\nu+\frac{1}{2}\right)\right\}\right],
\end{array}
$$


and

$$
\begin{gathered}
\frac{\Gamma\left(\nu+\frac{1}{2}\right)}{\Gamma(\nu+1)}=\sqrt{ } \pi C_{n}, \\
\psi(\nu+1)-\psi\left(\nu+\frac{1}{2}\right)=2\left(\log 2-1+\frac{1}{2}-\frac{1}{3}+\ldots+\frac{1}{2 \nu}\right)=2 R_{\nu,} \text { say ;* }
\end{gathered}
$$

with the special values $\quad \Gamma\left(\frac{1}{2}\right) / \Gamma(1)=\sqrt{ } \pi, \quad \psi(1)-\psi\left(\frac{1}{2}\right)=2 \log 2$.

Thus we obtain the approximate formula

$$
-\frac{1}{p}=(-1) \nu C_{\nu}\left(\frac{1}{t}-R_{\nu}\right)
$$

which applies also for the case $\nu=0$, if we write

$$
C_{0}=1, \quad R_{01}=\log 2 .
$$

'Po calculate the limiting form of equation (4), we can write $m+1=2 \nu$ in all the terms except the first, and the term in the bracket for which $n=\nu$; thus we gett

$$
\begin{aligned}
V_{1} & =\pi k a^{2 \nu}\left\{\frac{1}{\nu}-\sum_{1}^{\infty}(-1)^{n} \frac{C_{n}}{n-\nu}\left(\frac{v}{a}\right)^{2 n} P_{2 i n}\right\} \\
& +2 \pi k a^{2 \nu}(-1)^{\nu} C_{\nu} \lim _{t \rightarrow 0}\left\{\frac{1}{t}\left(\frac{r}{a}\right)^{2 \nu} P_{2 \nu}-\left(\frac{1}{t}-R_{\nu}\right)\left(\frac{r}{a}\right)^{2 \nu+t} P_{2 \nu+t}\right\},
\end{aligned}
$$

where for brevity we are now considering only the case when $\mu$ is positive; to deal with negative values of $\mu$, we need only change the finit conclu sions by writing $|\mu|$ for $\mu$.

Hence for the law of density $k s^{2 \nu-1}$, we obtain the formula for the potential of the disc at points for which $r<a$,

$$
\begin{aligned}
V_{1}= & \pi k a^{2 \nu}\left\{\frac{1}{\nu}-\sum_{1}^{\infty}(-1)^{n} \frac{C_{n}}{n-\nu}\left(\frac{r}{a}\right)^{2 n} P_{2 n}\right\} \\
& +2 \pi k a^{2 \nu}(-1)^{\nu} C_{\nu}\left\{R_{\nu}\left(\frac{r}{a}\right)^{2 \nu} P_{2 \nu}-Y_{2 \nu}\right\},
\end{aligned}
$$

where we write

$$
Y_{n}=\frac{\partial}{\partial n}\left\{\left(\frac{r}{a}\right)^{n} P_{n}\right\}
$$

An alternative form, equivalent to (6), will be found in (11) below.

- See, for instance, my book on Infinite Series, p. 475, Ex. 42.

$\dagger$ The symbol $\Sigma^{\prime}$ is used in the sense often adopted by Weierstrass and others; the accent implies that certain values of $n$ are excluded (here $n=\nu$ ) which would involve infinities in the summation. 
In the special case $\nu=0$, the formula (6) becomes

$$
V_{1}=\pi k \sum_{1}^{\infty}(-1)^{n-1} \frac{C_{n}}{n}\left(\frac{r}{a}\right)^{2 n} P_{2 n}+2 \pi k\left(\log 2-Y_{0}\right),
$$

which is the potential (when $r<a, \mu>0$ ) of a dise whose density is $k / s$.

We shall consider below more fully the form of the function $Y_{n}$, which does not appear to have presented itself previously. But before discussing this, it will be useful to remark that the limiting process which we have used can be avoided by writing $m=2 \nu-1$ in the integral. (2) for the potential. It will then be seen that the potential takes the form

$$
\begin{aligned}
\pi k a^{2 \nu} & \left(\frac{1}{\nu}-\sum_{1}^{\infty} \frac{(-1)^{n} C_{n}}{n-\nu}\left(\frac{r}{a}\right)^{2 n} P_{2 n}\right) \\
+2 \pi k r^{2 \nu} & {\left[-\frac{1}{2 \nu(2 \nu+1)}+\sum_{1}^{\infty} \frac{(-1)^{n} C_{n}(4 n+1)}{(2 n+2 \nu+1)(2 n-2 \nu)} P_{2 n}\right.} \\
& \left.+(-1)^{\nu} C_{\nu} P_{2 \nu}\left(\frac{1}{4 \nu+1}+\log \left(\frac{a}{r}\right)\right\}\right]
\end{aligned}
$$

The bracket in the last line takes the form $(-1)^{\nu} C_{\nu} P_{2 \nu} \log (a / r)+f(\theta)$; and so we are led to conjecture the existence of a solution of Laplace's equation which takes the form

$$
r^{n}\left\{P_{n} \log (a / r)+f_{1}(\theta)\right\},
$$

$n$ being an integer. We proceed next (in $\S$ II) to investigate the form of this solution, and we shall afterwards connect the solution with the function $Y_{n}$ defined in (7).

As a matter of fact the form of the potential was originally determined by the second process; but the limiting process has the advantage of indicating more clearly the relation of this case to the general formula (4).

\section{Investigation of certain Harmonics.}

The simplest case of these harmonics corresponds to (8), when we suppose $\nu=0$ or $m=1$; the integral (2) then leads to a harmonic of the form

$$
1+\log \left(\frac{a}{r}\right)+\sum_{1}^{\infty}(-1)^{n} C_{n} \frac{4 n+1}{2 n(2 n+1)} P_{2 n} .
$$

This series of zonals, as we shall prove in a moment, is equal to

$$
2 \log 2-1-\log (1+|\mu|,
$$


so that the harmonic is simply

$$
\log \left(\frac{4 a}{r+|z|}\right)
$$

which is at once seen to be a solution of Laplace's equation-it is, of course, a well-known solution, although not often wanted in mathematical physics.

To verify the identity quoted above, it is easiest to expand the function

$$
\log (1+|\mu|)
$$

in a series of zonal harmonics. It is readily seen that no odd harmonics can occur, and if we write

we find at once $\quad A_{0}=\frac{1}{2} \int_{-1}^{+1} \log (1+|\mu|) d \mu=\int_{11}^{1} \log (1+\mu) d \mu$

$$
\log (1+|\mu|)=A_{0}+\sum_{1}^{\infty} A_{n} P_{2_{n}}(\mu),
$$

Also

$$
\begin{aligned}
A_{n} & =\frac{4 n+1}{2} \int_{-1}^{+1} \log (1+|\mu|) P_{n n}(\mu) d \mu \\
& =(4 n+1) \int_{0}^{1} \log (1+\mu) P_{2 n}(u) d \mu .
\end{aligned}
$$

Thus using the differential equation for $P_{z_{n}}$, we find

$$
A_{n}=-\frac{4 n+1}{2 n(2 n+1)}\left[\log (1+\mu)\left(1-\mu^{2}\right) \frac{d P_{2 n}}{d \mu}\right]_{0}^{1}+\begin{gathered}
4 n+1 \\
2 n(2 n+1)
\end{gathered} \int_{0}^{1}(1-\mu) \frac{d P_{2 n}}{d \mu} d \mu .
$$

The integrated part is zero at both limits; and on integrating by parts again, we find

$$
A_{n}=\frac{4 n+1}{2 n(2 n+1)}\left[(1-\mu) P_{2 n}\right]_{n}^{1}+\frac{4 n+1}{2 n(2 n+1)} \int_{0}^{1} P_{2 n} d \mu
$$

Here the last term is zero, and so

$$
A_{n}=-\frac{4 n+1}{2 n(2 n+1)} P_{2 n}(0)=-\begin{gathered}
4 n+1 \\
2 n(2 n+1)
\end{gathered}(-1)^{n} C_{n},
$$

which confirms the result stated.

Returning to the formula (8), we now get the formula for the potential of a surface-density $k / s$, at points for which $r<a$,

$$
\pi k \sum_{1}^{\infty}(-1)^{n-1} \frac{C_{n}}{n}\left(\frac{r}{a}\right)^{2 n} P_{2 n}+2 \pi k \log \left(\frac{4 a}{r+|z|}\right)
$$

a result which is really the same as $(6 a)$.

To obtain the general case of the harmonics suggested by (8) we try to find a harmonic function of the form

$$
\mathrm{Y}_{n}=Z_{n} \log \left(\frac{r+z}{2 a}\right)+r^{n} f(u)
$$


the abbreviation $Z_{n}$ being used for $r^{n} P_{n}(\mu)$; it will be supposed here that $z$ and $\mu$ are positive.

Now $\Delta Z_{n}=0$, where $\Delta$ denotes Laplace's operator ; and as we have just seen

$$
\Delta \log \{(r+z) / 2 a\}=0,
$$

so that $\Delta\left\{Z_{n} \log \left(\frac{r+z}{2 a}\right)\right\}=2\left(\frac{\partial Z_{n}}{\partial x} \frac{x / r}{r+z}+\frac{\partial Z_{n}}{\partial y} \frac{y / r}{r+z}+\frac{\partial Z_{n}}{\partial z} \frac{1+z / r}{r+z}\right)$

$$
=\frac{2}{r+z}\left(\frac{n Z_{n}}{r}+\frac{\partial Z_{n}}{\partial z}\right) \text {. }
$$

Now it is easy to verify that

$$
\frac{\partial Z_{n}}{\partial z}=n Z_{n-1}
$$

and so we have the formula

$$
\Delta\left\{Z_{n} \log \left(\frac{r+z}{2 a}\right)\right\}=\frac{2 n}{1+\mu} r^{n-2}\left(P_{n}+P_{n-1}\right)
$$

Also

$$
\Delta\left\{r^{n} f(\mu)\right\}=r^{n-2}\left[n(n+1) f+\frac{d}{d \mu}\left\{\left(1-\mu^{2}\right) \frac{d f}{d \mu}\right\}\right],
$$

so that the condition $\Delta X_{n}=0$ leads to

$$
\frac{2 n}{1+\mu}\left(P_{n-1}+P_{n}\right)+n(n+1) f+\frac{d}{d \mu}\left\{\left(1-\mu^{2}\right) \frac{d f}{d \mu}\right\}=0 .
$$

It is easy to see that the first term in this formula is a polynomial of degree $(n-1)$, and that it is equal to twice*

$$
(2 n-1) P_{n-1}-(2 n-3) P_{n-2}+(2 n-5) P_{n-3}-\ldots+(-1)^{n-1} \text {. }
$$

We assume accordingly for $f$,

$$
f(u)=A_{n} P_{n}+A_{n-1} P_{n-1}+A_{n-2} P_{: 2-2}+\ldots+A_{0},
$$

- In fact, if the given expression is multiplied by $(1+\mu)$, we get for the product

$$
\begin{aligned}
n P_{n}+(2 n-1) P_{n-1} & +(n-1) P_{n-2} \\
-(n-1) P_{n-1} & -(2 n-3) P_{n-2}-(n-2) P_{n-2} \\
& +(n-2) P_{n-2}+(2 n-5) P_{n-3}+(n-3) P_{n-1} \\
=n P_{n}+n P_{n-1} . & +\ldots+
\end{aligned}
$$


and then our condition yields

$$
\begin{aligned}
2\left\{(2 n-1) P_{n-1}-(2 n-3)\right. & \left.P_{n-2}+\ldots+(-1)^{n-1} P_{0}\right\} \\
& +\sum_{s=0}^{n-1}\{n(n+1)-s(s+1)\} A_{s} P_{s}=0 .
\end{aligned}
$$

Hence $\quad A_{8}=(-1)^{n-s} \frac{(2 s+1)}{(n-s)(n+s+1)}, \quad$ for $\quad s=0,1, \ldots, n-1$;

and so, taking $A_{n}=0$ for simplicity, we find

$$
\begin{aligned}
f(\mu)=-2\left\{\frac{2 n-1}{1.2 n} P_{n-1}-\frac{2 n-3}{2(2 n-1)} P_{n-2}\right. & +\frac{2 n-5}{3(2 n-2)} P_{n-3}-\ldots \\
& \left.+(-1)^{n-1} \frac{1}{n(n+1)}\right\} .
\end{aligned}
$$

Thus our new solution of Laplace's equation takes the form

(9) $\quad X_{n}=r^{n} P_{n}(\mu) \log \left(\frac{r+z}{2 a}\right)$

$$
-2 r^{n}\left\{\frac{2 n-1}{1.2 n} P_{n-1}-\frac{2 n-3}{2(2 n-1)} P_{n-2}+\ldots+\frac{(-1)^{n-1}}{n(n+1)}\right\},
$$

and, of course, any arbitrary multiple of $r^{n} P_{n}(\mu)$ may be added to this without affecting the conclusion.

To obtain the solution commonly denoted by $r^{n} Q_{n}(\mu)$, we change the sign of $\mu$ in (9), and deduce, after division by $(-1)^{n}$, a second solution

(9a) $r^{n} P_{n}(\mu) \log \left(\frac{r-z}{2 a}\right)+2 r^{n}\left\{\frac{2 n-1}{1.2 n} P_{n-1}+\frac{2 n-3}{2(2 n-1)} P_{n-2}+\ldots+\frac{1}{n(n+1)}\right\}$.

Thus on subtracting (9a) from (9) and dividing by 2 , we get the solution

$$
\frac{1}{2},{ }^{n} P_{n}(\mu) \log \left(\frac{1+\mu}{1-\mu}\right)-2 r^{n}\left\{\frac{2 n-1}{1.2 n} P_{n-1}+\frac{2 n-5}{3(2 n-2)} P_{n-3}+\ldots\right\},
$$

which is one of the known formulæ for $r^{n} Q_{n}(\mu)$.

It will be seen that (9b) contains $r$ only through the power $r^{n}$; but, in contrast to this apparent advantage, there is the drawback that (9b) has an infinity when $\mu$ is equal to either -1 or +1 . On the contrary, (9) remains finite at $\mu=+1$, while $(9 a)$ is finite at $\mu=-1$; thus we should expect to find (9) of use on the positive side of the plane of $x y$, while $(9 a)$ will serve for points on the negative side of this plane.

In view of these calculations it is evident that when $\mu>0$, we can 
write the solution $Y_{n}$ of (7) in the form

$$
Y_{n}=\left\{X_{n}+A_{n} r^{n} P_{n}(\mu)\right\} / a^{n},
$$

where the coefficient $A_{n}$ is not easily found directly, but is obtained indirectly in (10) below.

III. Specification of Harmonics by their Values on the Axis of Symmetry.

It is useful to notice that the harmonics which we have been considering are completely specified by their values on the positive axis of symmetry (for which $\mu=1$ ). Thus the harmonic $r^{m+1} P_{m+1}(\mu)$ is represented by $r^{m+1}$ on the axis, whatever may be the value of $m$ (whether an integer or not).

Similarly the harmonic defined in (7) by the equation

$$
Y_{n}=\frac{\partial}{\partial n}\left\{\left(\frac{r}{a}\right)^{n} P_{n}(\mu)\right\}
$$

is represented on the axis by $(r / a)^{n} \log (r / a)$; while the function $X_{n}$, defined in (9) above, is represented by

$$
\begin{aligned}
& r^{n} \log \left(\frac{r}{a}\right)-2 r^{n}\left\{\frac{2 n-1}{1.2 n}-\frac{2 n-3}{2(2 n-1)}+\ldots+\frac{(-1)^{n-1}}{n(n+1)}\right\} \\
= & r^{n} \log \left(\frac{r}{a}\right)-2 r^{n}\left(1-\frac{1}{2}+\frac{1}{3}-\ldots-\frac{1}{2 n}\right) .
\end{aligned}
$$

Thus, in particular, we can obtain the relation between $X_{n}$ and $Y_{n}$ : on the axis we have the relation

$$
a^{n} Y_{n}-X_{n}=2 r^{n}\left(1-\frac{1}{2}+\frac{1}{3}-\ldots-\frac{1}{2 n}\right),
$$

and so, in general, we have the identity

$$
a^{n} Y_{n}=X_{n}+2 r^{n} P_{n}(u)\left(1-\frac{1}{2}+\frac{1}{3}-\ldots-\frac{1}{2 n}\right) \text {. }
$$

We shall obtain later (in $\S \mathrm{V}$ ) a direct proof of the relation (10); but it should be noted here that (10) leads to a formula equivalent to (6), for the potential of the disc with density $k s^{2 v-1}$, namely,

$$
\begin{aligned}
V_{1}= & \pi k a^{2 \nu}\left\{\frac{1}{\nu}-\stackrel{\Sigma}{\prime}_{1}^{\infty}(-1)^{n} \frac{C_{n}}{n-\nu}\left(\frac{r}{a}\right)^{2 n} P_{2 n}\right\} . \\
& +2 \pi k(-1)^{\nu} C_{\nu}\left\{r^{2 \nu} P_{2 \nu}\left(\log 2-S_{\nu}-2 S_{2 \nu}\right)-X_{2 \nu}\right\},
\end{aligned}
$$


where

$$
S_{\nu}=1-\frac{1}{2}+\frac{1}{3}-\ldots-\frac{1}{2 \nu}
$$

and

$$
S_{2 v}=1-\frac{1}{2}+\frac{1}{3}-\ldots-\frac{1}{4 \nu} .
$$

For instance, with $\nu=1$, we have

$$
S_{1}=\frac{1}{2}, \quad S_{2}=\frac{7}{12}, \quad S_{1}+2 S_{2}=\frac{5}{3} ;
$$

and so the potential corresponding to a density $k s$ is

$$
\begin{aligned}
V_{1}= & \pi k a^{2}\left\{1-\sum_{1}^{\infty}(-1)^{n} \frac{C_{n}}{n-1}\left(\frac{r}{a}\right)^{2 n} P_{2 n}\right\} \\
& -\pi k\left\{r^{2} P_{2}\left(\log 2-\frac{5}{3}\right)-X_{2}\right\},
\end{aligned}
$$

a result proposed in the Mathematical Tripos, Part II, 1909, and given in the abstract of this paper.

To illustrate the method of utilising the value of the potential on the axis to determine the potential generally, we may apply the process to a disc whose surface density is $k \mathrm{~s}^{\mathrm{in}}$. At a point on the axis, we have

Thus, taking $r>a$, we get

$$
V=2 \pi k \int_{0}^{n} \frac{s^{\prime \prime \prime+1} d s}{\sqrt{ }\left(s^{2}+r^{2}\right)} .
$$

$$
\begin{aligned}
V_{0} & =2 \pi k \int_{0}^{n} s^{m+1} d s\left\{\frac{1}{r}+\sum_{1}^{\infty}(-1)_{n} C_{n} \begin{array}{c}
s^{2 n} \\
\left.r^{2 n+1}\right\}
\end{array}\right. \\
& =2 \pi k a^{m+1}\left\{\frac{1}{m+2} \underset{v}{a}+\sum_{1}^{\infty}(-1)_{n} \frac{C_{n}}{2 n+m+2}\left(\frac{a}{r}\right)^{2 n+1}\right\},
\end{aligned}
$$

which leads at once to formula (1) above. But, if $r<a$, we have

$$
\begin{aligned}
V_{1}= & 2 \pi k \int_{0}^{r} s^{n+1} d s\left\{\frac{1}{r}+\sum_{1}^{\infty}(-1)^{n} C_{n} \frac{s^{2 n}}{r^{2 n+1}}\right\} \\
& +2 \pi k \int_{r}^{n} s^{m+1} d s\left\{\frac{1}{s}+\sum_{1}^{\infty}(-1)^{n} C_{n} \cdot \frac{r^{2 n}}{s^{2 n+1}}\right\} \\
= & 2 \pi k r^{\prime \mu+1}\left\{-\frac{1}{(m+1)(m+2)}+\sum_{1}^{\infty}(-1)^{n} C_{n} \frac{4 n+1}{(2 n+m+2)(2 n-m-1)}\right\} \\
& +2 \pi k a^{m+1}\left\{\frac{1}{m+1}-\sum_{1}^{\infty}(-1)^{n} \frac{C_{n}}{2 n-m-1}\left(\frac{r}{a}\right)^{2 n}\right\},
\end{aligned}
$$

a result which leads at once to (3) above.

It is not very easy, however, to apply a similar method, in general, to obtain (6); the formula obtained is substantially (8), but the coefficient of $r^{2 \nu} P_{\nu_{\nu}}$ is troublesome to express in a simple form. For the special cases $\nu=0, \nu=1$, we can proceed as follows.

- Of course the method is familiar (and goes back to Legendre) in the case when harmonics of integral order suffice to express the potential $V_{1}$. 
When $v=0$, we have

$$
\nabla=2 \pi \int_{0}^{\pi} \begin{gathered}
k d s \\
\sqrt{ }\left(s^{2}+r^{2}\right)
\end{gathered}=2 \pi k \sinh ^{-1}\left(\frac{a}{r}\right)=2 \pi k \log \left\{\frac{a+\sqrt{ }\left(a^{2}+r^{2}\right)}{r}\right\},
$$

and thus we find, * if $r<a$,

$$
V_{1}=2 \pi k\left\{\log \frac{2 a}{r}+\frac{1}{2} \frac{r^{2}}{2 a^{2}}-\frac{1.3}{2.4} \frac{r^{4}}{4 a^{4}}+\frac{1.3 .5}{2.4 .6} 6 \bar{r}^{6}-\ldots\right\},
$$

which leads to $(6 a)$ at once.

Similarly, when $\nu=1$, we find

$$
V=2 \pi k \int_{0}^{n}-\frac{s^{2} d s}{\sqrt{\left(s^{2}+r^{2}\right)}}=\pi k\left\{a \sqrt{ }\left(a^{2}+r^{2}\right)-r^{2} \sinh ^{-1}\left(\frac{a}{r}\right)\right\},
$$

and expanding similarly we obtain the special case of (6) which corresponds to $\nu=1$; the terms of special interest here are those in $r^{2}$, which are seen to be

$$
\pi k\left\{\frac{1}{2} r^{2}-r^{2} \log (2 a / r)\right\},
$$

and these lead to the general values

$$
\pi k\left\{a^{2} Y_{2}-\left(\log 2-\frac{1}{2}\right) r^{2} P_{2}\right\} .
$$

IV. The Solutions Inverse to $X_{n}$.

Professor Hobson pointed out to me, in 1909, that the solutions $X_{n}$ can be derived from known solutions as follows.

It is a familiar fact that if $f(x, y, z)$ is a solution of Laplace's equation, then another solution can be derived by inversion, which leads to the form

$$
\frac{1}{r} f\left(\frac{x}{r^{2}}, \frac{y}{r^{2}}, \frac{z}{r^{2}}\right) \text {. }
$$

Now $\log \frac{1}{2}(v+z)$ is a known solution t and consequently we have another

$$
\frac{1}{r} \log \left(\frac{r+z}{2 r^{2}}\right)
$$

From this we can derive others by differentiation in the form

$$
\begin{aligned}
& \frac{(-1)^{n}}{n !} \frac{\partial^{n}}{\partial z^{n}}\left\{\frac{1}{r} \log \left(\frac{r+z}{2 r^{2}}\right)\right\} \\
& =\frac{P_{n}(\mu)}{r^{n+1}} \log \left(\frac{r+z}{2 r^{2}}\right) \\
& \quad+\left\{-\frac{P_{n-1}(\mu)}{r^{n}}\left(\frac{1}{r}-\frac{2 z}{r^{2}}\right)+\frac{1}{2 !} \frac{P_{n-2}(\mu)}{r^{n-1}}\left(-\frac{z}{r^{3}}-\frac{2}{r^{2}}+\frac{4 z^{2}}{r^{4}}\right)-\ldots\right\},
\end{aligned}
$$

- See, for example, my book, Infinite Series, p. 163, Ex. 14.

+ Actually $\log (r+z)=\int \frac{d z}{r}$, so that this is an obvious solution. 
by applying Leibnitz's theorem on repeated differentiation. Now this expression is of the form

$$
\frac{P_{n}(\mu)}{r^{n+1}} \log \left(\frac{r+z}{2 r^{2}}\right)+\frac{f_{n}(\mu)}{r^{n+1}},
$$

where $f_{n}$ is a polynomial of degree $n$ in $\mu$. Inverting again, we derive a solution of the form

$$
r^{n} P_{n}(\mu) \log \frac{1}{2}(r+z)+r^{n} f_{n}(\mu) .
$$

To actually obtain a formula for $f_{n}(\mu)$ it is, however, easier to apply the differential equation directly to the function (13); and so we return substantially to the calculations given in $\$$ II above. The actual relation of this harmonic to $X_{n}$ and $Y_{n}$ can be determined by examining its form at. points on the axis, for which (12) becomes

$$
\frac{(-1)^{n}}{n !} \frac{d^{n}}{d r^{n}}\left\{\frac{1}{r} \log \left(\frac{1}{r}\right)\right\}=\frac{1}{r^{n+1}}\left(\log \frac{1}{r}+1+\frac{1}{2}+\ldots+\frac{1}{n}\right) .
$$

Consequently (13) becomes on the axis

$$
r^{n}\left(\log r+1+\frac{1}{2}+\ldots+\frac{1}{n}\right),
$$

and (13) is therefore (in general) equal to

$$
Y_{n}+r^{n} P_{n}(\mu)\left(1+\frac{1}{2}+\ldots+\frac{1}{n}\right),
$$

where we have taken $a=1$ in the definition of $Y_{n}$.

\section{V. $A$ direct Determination of a Formula for $Y_{n}$.}

To abbreviate the formulæ, put $a=1$ in the definition (7), and then

$$
Y_{n}=\frac{\partial}{\partial n}\left(r^{n} P_{n}\right)=r^{n}\left(P_{n} \log r+\frac{\partial P_{n}}{\partial n}\right) .
$$

To proceed further we shall use the complex integral

$$
P_{n}(\mu)=\frac{1}{2 \pi \iota} \int \frac{t^{n} d t}{\sqrt{ }\left(1-2 \mu t+t^{2}\right)} .
$$

In order to make the integrand single-valued we make cuts in the $t$-plane, along the negative half of the real axis, and along the line which joins the branch-points $A, B$ given by

$$
1-2 \mu t+t^{2}=0
$$

or by $\quad t=\mu+\imath \sqrt{ }\left(1-\mu^{3}\right)$ and $\mu-\imath \sqrt{ }\left(1-\mu^{2}\right)$.

8MR. 2. vor. 12 . No. 1166 . 
We assume that $\mu$ is positive, and this is in agreement with the applicstions which have been made of the function $Y_{n}$.

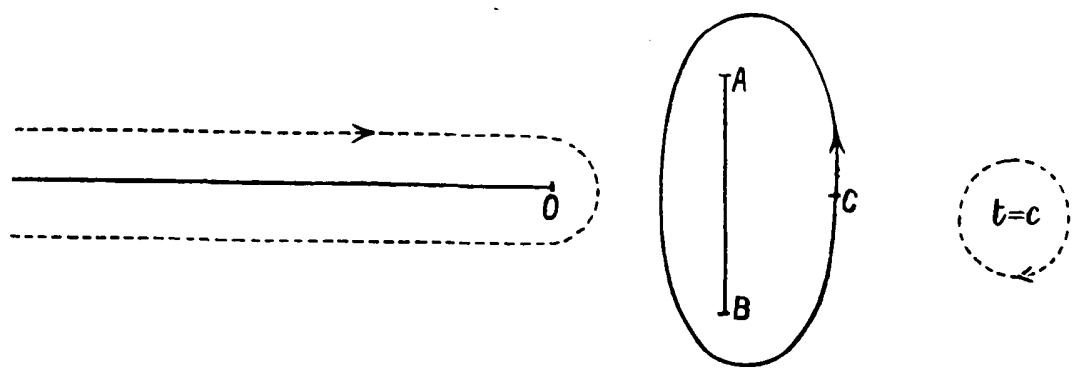

Further, we suppose that the integrand is real and positive at the point $C$ in which the path of integration cuts the real axis, on the side of $A B$ which is further from the origin; with this choice of integrand it will be seen that the integral (15) gives the value of $P_{n}(\mu)$.

A simple proof may be given as follows. Let the integral be called $I_{n}$, and then write $t=v / r$. We get

$$
r^{n} I_{n}=\frac{1}{2 \pi \imath} \int_{\sqrt{ }\left(r^{2}-2 v z+v^{2}\right)}^{v^{n} d v}
$$

where the integral extends round a similar path in the $v$-plane. But

$$
r^{2}-2 v z+v^{2}=x^{2}+y^{2}+(z-v)^{2},
$$

so that $\left(r^{2}-2 v z+v^{2}\right)^{-1}$ satisfies Laplace's equation. Thus the same is true of $r^{n} I_{n}$, and so $I_{n}$ satisfies Legendre's equation in $\mu$. To identify $I_{n}$ with $P_{n}$, we consider the value of $I_{n}$ when $\mu=1$. We have then

$$
I_{n}=\frac{1}{2 \pi t} \int \frac{t^{n} d t}{t-1}
$$

where the path surrounds $t=1$, and $t^{n}$ is to be real and positive at the point $C$ (since $t-1$ is there positive); and thus $I_{n}$ is now seen to be equal to 1 , when $\mu=1$. Hence

$$
I_{n}=P_{n}(\mu) \text {, }
$$

because $P_{n}(\mu)$ is the only solution of Legendre's equation which reduces to unity at $\mu=1$.

It now follows that

$$
\frac{\partial P_{n}}{\partial n}=\frac{1}{2 \pi \iota} \int \frac{t^{n} \log t d t}{\sqrt{ }\left(1-2 \mu t+t^{2}\right)},
$$

taken round the same path as (15); and so if $|c|>1$, we can write

$$
\sum_{1}^{\infty} \frac{\partial P_{n}}{\partial n} \frac{1}{c^{n+1}}=\frac{1}{2 \pi \iota} \int \frac{\log t d t}{(c-t) \sqrt{ }\left(1-2 \mu t+t^{2}\right)} .
$$

The last integrand is, when $|t|$ is large, of the order $(\log t) / t^{2}$, so that the value of the integral taken round any portion of a large circle tends to 
zero as the radius tends to infinity; and thus the original path of integration can be transformed into the dotted paths indicated in the diagram.

From the circle round $t=c$, we get*

$$
(\log c) / \sqrt{ }\left(1-2 \mu c+c^{2}\right)=(\log c) / q, \text { say; }
$$

where the square root $q$ will be real and positive, if $c$ is real and greater than 1, as we shall suppose for simplicity of statement.

On the part of the dotted path which lies above the negative axis, we have (in the limiting form of the path)

$$
t=-x, \quad \log t=\log x+\pi \iota, \quad \sqrt{ }\left(1-2 \mu t+t^{2}\right)=-\sqrt{ }\left(1+2 \mu x+x^{2}\right),
$$

and so this part of the integral yields

$$
+\frac{1}{2 \pi \iota} \int_{\infty}^{0} \frac{(\log x+\pi \iota) d x}{(c+x) \sqrt{ }\left(1+2 \mu x+x^{2}\right)}=-\frac{1}{2 \pi \iota} \int_{0}^{\infty} \frac{(\log x+\pi \iota) d x}{(c+x) \sqrt{ }\left(1+2 \mu x+x^{2}\right)} .
$$

For the path below the negative axis

$$
t=-x, \quad \log t=\log x-\pi \iota, \quad \sqrt{ }\left(1-2 \mu t+t^{2}\right)=-\sqrt{ }\left(1+2 \mu x+x^{2}\right),
$$

and so this part of the integral gives

$$
+\frac{1}{2 \pi \iota} \int_{0}^{\infty} \frac{(\log x-\pi \iota) d x}{(c+x) \sqrt{ }\left(1+2 \mu x+x^{2}\right)}
$$

Thus on combination, we get, from (16), the equation

$$
\sum_{0}^{\infty} \frac{1}{c^{n+1}} \frac{\partial P_{n}}{\partial n}=\frac{\log c}{q}-\int_{0}^{\infty} \frac{d x}{(c+x) \sqrt{ }\left(1+2 \mu x+x^{2}\right)} .
$$

The last integral is easily evaluated by elementary methods and is equal tot

so that

$$
\frac{2}{q} \tanh ^{-1}\left(\frac{q}{1+c}\right)
$$

$$
\sum_{0}^{\infty} \frac{1}{c^{n+1}} \frac{\partial P_{n}}{\partial n}=\frac{1}{q}\left\{\log c-2 \tanh ^{-1}\left(\frac{q}{1+c}\right)\right\}=\frac{U}{q}, \text { say. }
$$

* The fact that the circle is described in the clockwise sense just neutralizes the change of sign introduced by our having $(c-t)$ in place of $(t-c)$ in the denominator of (16).

$\dagger$ See, for instance, Messenger of Mathematics, Vol. 35, p. 1.31. 
Then, if we differentiate out the bracket, we find

$$
\begin{aligned}
\frac{\partial U}{\partial c} & =\frac{1}{c}-\frac{2}{q} \frac{(1+c)(c-\mu)-\left(1-2 \mu c+c^{2}\right)}{(1+c)^{2}-\left(1-2 \mu c+c^{2}\right)} \\
& =\frac{1}{c}-\frac{c-1}{c} \frac{1}{q} \\
& =\sum_{1}^{\infty} \frac{1}{c^{n+1}}\left(P_{n-1}-P_{n}\right) .
\end{aligned}
$$

Hence

$$
U=U_{0}+\sum_{1}^{\infty} \frac{1}{n c^{n}}\left(P_{n}-P_{n-1}\right)
$$

where $U_{0}$ is the limit of $U$ when $c$ tends to infinity. But

$$
U=\log \frac{c(1+c-q)}{1+c+q}=\log \frac{c .2 c(1+\mu)}{(1+c+q)^{2}},
$$

so that

$$
U_{0}=\log \frac{1}{2}(1+\mu)
$$

and

$$
U=\log \frac{1}{2}(1+\mu)+\sum_{1}^{\infty} \frac{1}{n c^{n}}\left(P_{n}-P_{n-1}\right) .
$$

If we multiply the last series by

$$
\frac{1}{q}=\frac{1}{c}+\sum_{1}^{\infty} \frac{1}{c^{n+1}} P_{n}
$$

we obtain, from (17), the formula*

$$
\begin{gathered}
\frac{\partial P_{n}}{\partial n}=P_{n} \log \frac{1}{2}(1+\mu)+P_{n-1}\left(P_{1}-P_{0}\right)+\frac{1}{2} P_{n-2}\left(P_{2}-P_{1}\right)+\ldots \\
+\frac{1}{n}\left(P_{n}-P_{n-1}^{\mathrm{e}}\right) .
\end{gathered}
$$

It is clear that the last part of (18) is a polynomial of degree $n$ in $\mu$, and so can be arranged in the form

$$
A_{n} P_{n}+A_{n-1} P_{n-1}+\ldots+A_{0} P_{0},
$$

but it is not easy to obtain the form of these coefficients $A_{8}$ directly; although one relation is evident by writing $\mu=1$, namely,

$$
A_{n}+A_{n-1}+\ldots+A_{0}=0,
$$

a result which is also confirmed by the fact that $P_{n}(1)=1$ for all values of $n$, so that, when $\mu$ is 1 ,

$$
\frac{\partial P_{n}}{\partial n}=0
$$

- In the special case $n=0$, the value of the differential coefficient reduces to $\log \frac{2}{2}(1+\mu)$; this special case was obtained by a different method in 1906 (Proc. London Math. Soc., Ser, 2, Vol, 4, p. 222, footnote). 
On substituting from (18) in (14), we see that

$$
Y_{n}=r^{n}\left\{P_{n} \log \frac{1}{2}(r+z)+A_{n} P_{n}+A_{n-1} P_{n-1}+\ldots+A_{0} P_{0}\right\},
$$

and since $Y_{n}$ satisfies Laplace's equation, it follows from $\S$ II, that

$$
\begin{aligned}
& A_{n-1}=-2\left(\frac{2 n-1}{1.2 n}\right)=-2\left(1-\frac{1}{2 n}\right), \\
& A_{n-2}=+2\left(\frac{2 n-3}{2(2 n-1)}\right)=+2\left(\frac{1}{2}-\frac{1}{2 n-1}\right), \\
& A_{n-3}=-2\left(\frac{2 n-5}{3(2 n-2)}\right)=-2\left(\frac{1}{3}-\frac{1}{2 n-2}\right), \quad \& c ., \\
& A_{0}=(-1)^{n} \frac{2}{n(n+1)}=(-1)^{n} 2\left(\frac{1}{n}-\frac{1}{n+1}\right) .
\end{aligned}
$$

Thus, from (19), we have the result

$$
A_{n}=-\left(A_{n-1}+A_{n-2}+\ldots+A_{0}\right)=2\left(1-\frac{1}{2}+\frac{1}{3}-\frac{1}{4}+\ldots-\frac{1}{2 n}\right),
$$

a result which we found before in $\S$ III by comparison of the values of $Y_{n}$ and $X_{n}$ on the axis of symmetry.

VI. Potential of a Hemisphere, with a Law of Density $k s^{m-1}$, where $s$ is the Distance from the Centre.

We take the base of the hemisphere as the plane of $x y$, the centre as the origin, and the hemisphere as lying on the positive side of the plane. Then the potential of the hemisphere is

$$
V=\int_{0}^{a} k s^{m+1} d s \int_{0}^{l \pi} \sin \theta^{\prime} d \theta^{\prime} \int_{0}^{2 \pi} \frac{d \phi}{\sqrt{\left(r^{2}+s^{2}-2 r s \cos \gamma\right)}},
$$

where

$$
\cos \gamma=\cos \theta^{\prime} \cos \theta+\sin \theta^{\prime} \sin \theta \cos \phi \text {. }
$$

Now

$$
\int_{0}^{2 \pi} P_{n}(\cos \gamma)=2 \pi P_{n}\left(\cos \theta^{\prime}\right) P_{n}(\cos \theta)
$$

and

$$
\int_{0}^{3 \pi} \sin \theta^{\prime} d \theta^{\prime} P_{n}\left(\cos \theta^{\prime}\right)=\int_{0}^{1} P_{n}\left(\mu^{\prime}\right) d \mu^{\prime},
$$

so that the value of this integral will be zero if $n$ is even. Also we have

$$
\int_{0}^{1} P_{0}\left(\mu^{\prime}\right) d \mu^{\prime}=1, \quad \int_{0}^{1} P_{2 n+1}\left(\mu^{\prime}\right) d \mu^{\prime}=(-1)^{n} K_{n},
$$

if

$$
K_{n}=\frac{1.3 \ldots(2 n-1)}{2.4 \ldots(2 n+2)} \quad \text { and } \quad K_{0}=\frac{1}{2} .
$$


These integrals are readily obtained by integrating the series

$$
\left.\frac{1}{\sqrt{ }\left(1-2 \mu^{\prime} t+t^{2}\right)}=1+\sum_{1}^{\infty} P_{n}\left(\mu^{\prime}\right) t^{\prime \prime} \quad \text { (where }|t|<1\right),
$$

from $\mu^{\prime}=0$ to $\mu^{\prime}=1$; and this gives

$$
1+\sum_{1}^{\infty} t^{n} \int_{0}^{1} P_{n}\left(\mu^{\prime}\right) d \mu^{\prime}=\frac{1}{t}\left\{\sqrt{ }\left(1+t^{2}\right)-(1-t)\right\}=1+\frac{1}{2} t+\sum_{1}^{x}(-t)^{n} K_{n} t^{2 n+1},
$$

from which the results stated are obvious.

If now $r>a$, we can expand the square root in (20) in the form

$$
\frac{1}{r}+\sum_{1}^{\infty} \frac{s^{n}}{r^{n+1}} P_{n}(\cos \gamma)
$$

and so, when $r>a$, we find the potential of the bemisphere,

$$
V_{0}=2 \pi k a^{m+1}\left\{\frac{1}{m+2} \frac{a}{r}+\sum_{0}^{\infty}(-1)^{n} \frac{K_{n}}{2 n+m+3}\left(\frac{a}{r}\right)^{2 n+2} P_{2 n+1}(u)\right\}
$$

It is perhaps worth while noting that if we write

$$
V_{0}=F(r, \mu)
$$

then

$$
F(r, \mu)+F(r,-\mu)=\frac{4 \pi k a^{m+2}}{(m+2) r},
$$

which is the potential of a complete sphere having the same law of density.

We now return to the case $r<a$; for which (as in the case of the dises) we must divide the integral with respect to $s$ into two parts, from 0 to $r$ and from $r$ to $a$, and then use the two different series

$$
\frac{1}{r}+\sum_{1}^{\infty} \frac{s^{n}}{r^{n+1}} P_{n}(\cos \gamma) \text { and } \frac{1}{s}+\sum_{1}^{\infty} \frac{r^{n}}{s^{n+1}} P_{n}(\cos \gamma)
$$

in these two parts.

After carrying out the integrations and rearranging the series in the same way as (3), we get*

$$
\begin{aligned}
V_{1} \text { or } V_{2}= & 2 \pi k r^{m+1}\left\{-\frac{1}{(m+1)(m+2)}\right. \\
& \left.\quad+\sum_{0}^{\infty}(-1)^{n} \frac{(4 n+3) K_{n}}{(2 n+m+3)(2 n-m)} P_{2 n+1}(\mu)\right\} \\
& +2 \pi k a^{n+1}\left\{\frac{1}{m+1}-\sum_{0}^{\infty}(-1)^{n} \frac{K_{n}}{2 n-m}\left(\frac{r}{a}\right)^{2 n+1} P_{2 n+1}(\mu)\right\}
\end{aligned}
$$

- It will be necessary to distinguish between the potentials on the positive and negative sides of the base; and we denote by $V_{1}$ the potential when $\mu>0$, by $V_{2}$ the potential when $\mu<0$, so that $V_{1}$ refers to points inside the hemisphere and $V_{2}$ to external points. 
Now since $V_{2}$ satisfies Laplace's equation it is suggested that the coefficient of $r^{m+1}$ will be proportional to $P_{m+1}(-\mu)$ when $\mu$ is negative.

To investigate this conjecture we shall find the coefficients $L_{n}$, so that

and

$$
\sum_{0}^{\infty} L_{n} P_{2 n+1}(\mu)=P_{m+1}(\mu)-P_{m+1}(0) \quad(\mu>0),
$$

$$
=P_{m+1}(0)-P_{m+1}(-\mu) \quad(\mu<0) \text {. }
$$

The function so defined is clearly an odd function of $\mu$, which is zero at $\mu=0$, and hence is expressible as a series of odd harmonics only. Thus we have

$$
\frac{2 L_{n}}{4 n+3}=2 \int_{0}^{1}\left\{P_{m+1}(\mu)-P_{m+1}(0)\right\} P_{2 n+1}(\mu) d \mu
$$

Now Legendre's equation gives

$$
\begin{aligned}
\{(2 n+1)(2 n+2)-(m+1)(m+2)\} & \int_{0}^{1} P_{m+1}(\mu) P_{2 n+1}(\mu) d \mu \\
& =\left[\left(1-\mu^{2}\right)\left\{P_{2 n+1}(\mu) \frac{d P_{m+1}}{d \mu}-P_{m+1}(\mu) \frac{d P_{2 n+1}}{d \mu}\right\}\right]_{0}^{\mathrm{T}} \\
& =P_{m+1}(0)\left(\frac{d P_{2 n+1}}{d \mu}\right)_{0}
\end{aligned}
$$

But

$$
\left(1-\mu^{2}\right) \frac{d P_{2 n+1}}{d \mu}=(2 n+1)\left(P_{2 n}-\mu P_{2 n+1}\right),
$$

so that

$$
\left(\frac{d P_{2 n+1}}{d \mu}\right)_{0}=(2 n+1) P_{2 \mu}(0)=(-1)^{n} \frac{1.3 \ldots(2 n+1)}{2.4 \ldots 2 n}
$$

$$
=(-1)^{n}(2 n+1)(2 n+2) K_{n} \text {. }
$$

Hence $\quad \int_{0}^{1} P_{m+1}(\mu) P_{2 n+1}(\mu) d \mu=(-1)^{n} \frac{(2 n+1)(2 n+2)}{(2 n+1)(2 n+2)-(n+1)(m+2)} K_{n} P_{m+1}(0)$,

and similarly $\quad \int_{0}^{1} P_{m+1}(0) P_{2 n+1}(\mu) d \mu=(-1)^{n} K_{n} P_{m-1}(0)$.

Thus $\quad \int_{0}^{1}\left\{P_{n+1}(\mu)-P_{m+1}(0)\right\} P_{2 n+1}(\mu) d \mu=(-1)^{n} \frac{(m+1)(m+2) K_{n} P_{m+1}(0)}{(2 n-m)(2 n+m+3)}$,

and so we find $\quad L_{n}=(-1)^{n} \frac{(4 n+3) K n}{(2 n-m)(2 n+n+3)}(m+1)(m+2) P_{m+1}(0)$.

Hence we have the formulæ

$$
\begin{aligned}
\sum_{0}^{\infty} \frac{(-1)^{n}(4 n+3) K_{n}}{(2 n-m)(2 n+m+3)} P_{2 n+1}(\mu)= & \frac{P_{m+1}(\mu)-P_{m+1}(0)}{(m+1)(m+2) P_{m+1}(0)}, \text { if } \mu>0, \\
& \frac{P_{m+1}(0)-P_{m+1}(-\mu)}{(m+1)(m+2) P_{m+1}(0)}, \text { if } \mu<0 .
\end{aligned}
$$

or

'Thus, on substituting in (22), we get the potentials, when $r<a$,

$$
\begin{aligned}
& V_{1}=\frac{2 \pi k r^{m+1}}{(m+1)(m+2)}\left\{-2+\frac{P_{m+1}(\mu)}{P_{m+1}(0)}\right\}+F(r, \mu), \\
& V_{2}=\frac{2 \pi k r^{m+1}}{(m+1)(m+2)}\left\{-\frac{P_{m+1}(-\mu)}{P_{m+1}(0)}\right\}+F(r, \mu),
\end{aligned}
$$


where

$$
F(r, \mu)=2 \pi k a^{m+1}\left\{\frac{1}{m+1}-\sum_{0}^{\infty}(-1)^{n} \frac{K_{n}}{2 n-m}\left(\frac{r}{a}\right)^{2 n+1} P_{2 n+1}(\mu)\right\} .
$$

These formulæ carry with them certain obvious means of verification; thus we get

$$
\Delta V_{1}=-4 \pi k r^{n-1}, \quad \Delta V_{2}=0,
$$

in agreement with Poisson's and Laplace's equations. Again, at the base of the hemisphere $(\mu=0)$, we have

$$
V_{1}=V_{2}, \quad \frac{1}{r} \frac{\partial V_{1}}{\partial \mu}=\frac{1}{r} \frac{\partial V_{2}}{\partial \mu},
$$

which should be the case in view of the general properties of the potential of a solid.

As an illustration, we may consider the case of a uniform hemisphere, with $m=1$; here

and so

$$
P_{m+1}(\mu)=\frac{1}{2}\left(3 \mu^{2}-1\right), \quad P_{m+1}(0)=-\frac{1}{3},
$$

and so

$$
\begin{aligned}
& V_{1}=-\frac{1}{3} \pi k r^{2}\left(1+3 \mu^{2}\right)+F(r, \mu), \\
& V_{2}=-\frac{1}{3} \pi k r^{2}\left(1-3 \mu^{2}\right)+F(r, \mu),
\end{aligned}
$$

where

$$
F(r, \mu)=2 \pi k a^{2}\left\{\frac{1}{2}-\sum_{0}^{\infty}(-1)^{n} \frac{K_{n}}{2 n-1}\left(\frac{r}{a}\right)^{2 n+1} P_{2 n+1}(\mu)\right\} .
$$

Expressed in terms of $x, y, z$, these functions take the forms (as far as terms of the second degree)

$$
\begin{aligned}
& V_{1}=\pi k\left\{a^{2}+a z-\frac{1}{3}\left(x^{2}+y^{2}+4 z^{2}\right)\right\}, \\
& V_{2}=\pi k\left\{a^{2}+a z-\frac{1}{3}\left(x^{2}+y^{2}-2 z^{2}\right)\right\},
\end{aligned}
$$

results originally given, I believe, by Mr. R. R. Webb.

When $m$ is an even integer $(2 \nu)$, the formulæ (23), (24) require modification; and the most direct method is to apply again the same limiting process as in $\S 1$ above by writing $m=2 \nu+t$, and making $t$ tend to zero. Now we proved on p. 104 that

$$
(m+2) P_{n+1}(0)=\frac{2}{\sqrt{ } \pi} \cos \left\{\frac{1}{2}(m+1) \pi\right\} \frac{\Gamma\left(\frac{1}{2} m+2\right)}{\Gamma\left(\frac{1}{2} m+\frac{3}{2}\right)},
$$

and so we find

$$
(m+1)(m+2) P_{m+1}(0)=-\frac{4}{\sqrt{ } \pi} \sin \left(\frac{1}{2} m \pi\right) \frac{\Gamma\left(\frac{1}{2} m+2\right)}{\Gamma\left(\frac{1}{2} m+\frac{1}{2}\right)} .
$$

Thus $\frac{1}{(m+1)(m+2) P_{m+1}(0)}=(-1)^{\nu-1} \frac{\sqrt{ } \pi}{4 \sin \left(\frac{1}{2} t \pi\right)} \frac{\Gamma\left(\nu+\frac{1}{2}+\frac{1}{2} t\right)}{\Gamma\left(\nu+2+\frac{1}{2} t\right)}$. 
and, approximating as on pp. 104, 105, the last formula becomes

because

$$
(-1)^{\nu-1} \frac{1}{t} K_{\nu}\left[1+\frac{1}{2} t\left\{\psi\left(\nu+\frac{1}{2}\right)-\psi(\nu+2)\right\}\right],
$$

Also

$$
\frac{1}{2 \sqrt{ } \pi} \frac{\Gamma\left(\nu+\frac{1}{2}\right)}{\Gamma(\nu+2)}=\frac{1.3 \ldots(2 \nu-1)}{2.4 \ldots(2 \nu+2)}=K_{\nu} .
$$

where, as before, $\quad R_{\nu}=\log 2-\left(1-\frac{1}{2}+\frac{1}{3}-\ldots-\frac{1}{2 \nu}\right)$.

Thus, to the necessary degree of accuracy, we can write in (23) and (24),

$$
\frac{1}{(m+1)(m+2) P_{m+1}(0)}=(-1)^{\nu-1} K_{\nu}\left(\frac{1}{t}-R_{\nu}-\frac{1}{2 \nu+2}\right) \text {. }
$$

And now, on taking the limit as $t$ tends to zero, we obtain the potential of a hemisphere whose density is $k s^{2 \nu-1}$, in the form

$$
\begin{aligned}
V_{1}= & -\frac{2 \pi k r^{2 v+1}}{(2 \nu+1)(\nu+1)}+G(r, \mu) \\
& +2 \pi k a^{2 \nu+1}(-1)^{\nu-1} K_{v}\left\{Y_{2 \nu+1}-\left(R_{\nu}+\frac{1}{2 \nu+2}\right)\left(\frac{r}{a}\right)^{2 \nu+1} P_{2 \nu+1}(\mu)\right\},
\end{aligned}
$$

(27) $V_{2}=G(r, \mu)$

$$
+2 \pi k a^{2 \nu+1}(-1)^{\nu-1} K_{\nu}\left\{-Y_{2 \nu+1}-\left(R_{\nu}+\frac{1}{2 \nu+2}\right)\left(\frac{v}{a}\right)^{2 \nu+1} P_{2 \nu+1}(\mu)\right\},
$$

where

$$
G(r, \mu)=\pi k a^{2 \nu+1}\left\{\frac{2}{2 \nu+1}-\sum_{0}^{\infty}(-1)^{n} \frac{K_{n}}{n-\nu}\left(\frac{r}{a}\right)^{\frac{2 n+1}{2}} P_{2 n+1}(\mu)\right\} .
$$

In (27) the value of $Y_{2 v+1}(|\mu|)$ is to be used; in fact, the function $Y_{u}$ has been defined only for positive values of the argument.

As an illustration, let us take the case $\nu=0$, which corresponds to the law of density $k / s$. Then we get

and

$$
R_{0}=\log 2 \text {, }
$$

or

$$
\begin{aligned}
a Y_{1}= & z \log \left(\frac{r+z}{2 a}\right)-r+z, \text { if } z>0 \\
& -z \log \left(\frac{r-z}{2 a}\right)-r-z, \text { if } z<0
\end{aligned}
$$

so that our formulæ reduce to

$$
\begin{aligned}
& V_{1}=2 \pi k(a-r)+\pi k\left\{z \log \left(\frac{4 a}{r+z}\right)+r-\frac{1}{2} z j+G_{1},\right. \\
& V_{2}=2 \pi k a+\pi k\left\{z \log \left(\frac{4 a}{r-z}\right)-r-\frac{1}{2} z\right\}+G_{1},
\end{aligned}
$$


where

$$
\begin{aligned}
G_{1} & =\pi k a \sum_{0}^{\infty}(-1)^{n-1} \frac{K_{n}}{n}\left(\frac{r}{a}\right)^{m+n+1} P_{2 ! n+1} \\
& =\pi k a !\left(\frac{1}{2.4}\left(\frac{r}{a}\right)^{3} P_{3}-\frac{1.3}{2.4 .6} \frac{1}{2}\left(\frac{r}{a}\right)^{5} P_{5}+\ldots\right) .
\end{aligned}
$$

As a verification it is not difficult to calculate directly the potential on the axis, which is

or

$$
\begin{aligned}
& V_{1}=\left.2 \pi\right|_{n} ^{n} k s d s \int_{0}^{l \pi} \frac{\sin \theta^{\prime} d \theta^{\prime}}{\sqrt{\left(r^{2}-2 r s \cos \theta^{\prime}+s^{2}\right)^{\prime}}}, \\
& V_{2}=2 \pi \int_{0}^{a} k s d s \int_{0}^{1 \pi} \frac{\sin \theta^{\prime} d \theta^{\prime}}{\sqrt{ }\left(r^{2}+2 r s \cos \theta^{\prime}+s^{2}\right)} .
\end{aligned}
$$

Hence we find

$$
\begin{aligned}
V_{1} & =\frac{2 \pi k}{r} \int_{0}^{a}\left\{\sqrt{ }\left(r^{2}+s^{2}\right)-|r \cdots s|\right\} d s \\
& =\frac{\pi k}{r}\left\{a \sqrt{ }\left(r^{3}+a^{2}\right)+r^{2} \sinh ^{-1}\left(\begin{array}{c}
a \\
r \\
r
\end{array}\right)-r^{2}-(a-r)^{2}\right\},
\end{aligned}
$$

and

$$
\begin{aligned}
V_{2} & =\frac{2 \pi k}{r} \int_{0}^{\prime \prime}\left\{(r+s) \quad \sqrt{ }\left(r^{2}+s^{2}\right)\right\} d s \\
& =\frac{\pi k}{r}\left\{(a+r)^{2}-r^{2}-a \sqrt{ }\left(r^{2}+a^{2}\right)-r^{2} \sinh ^{-1}\left(\frac{a}{r}\right)\right\} .
\end{aligned}
$$

We can then expand in powers of $r / a$, using the series quoted on p. 112 for $\sinh ^{-1}(a / r)$, which leads to the results (on the axis)

$$
\begin{aligned}
& V_{1}=2 \pi k(a-r)+\pi k r\left\{\log (2 a / r)+\frac{1}{2}\right\}+H(r), \\
& V_{:}=2 \pi k a-\pi k r\left\{\log (2 a / r)+\frac{1}{2}\right\}-H(r),
\end{aligned}
$$

where

$$
H(r)=\pi k a\left\{\frac{1}{2.4}\left(\frac{r}{a}\right)^{3}-\frac{1.3}{2.4 .6} \frac{1}{2}\left(\frac{r}{a}\right)^{5}+\frac{1.3 .5}{2.4 .6 .8}-\frac{1}{3}\left(\frac{r}{a}\right)^{7}-\ldots\right\} .
$$

These harmonize with the results obtained from our general formulæ.

\section{Appendix (January, 1913).}

Since the foregoing pages were written out I have had the opportunity of seeing Mr. G. N. Watson's formulæ, which were published in the Abstracts of the November meeting of the Society.

It is there remarked that (in the case of discs) the formulæ for the potentials $V_{1}, V_{0}$ along the axis of symmetry can be converted into one another by the use of complex integrals containing Gamma functions.

By a slight modification it appears to be easy to utilise this method, not only on the axis, but for any point in space; and the process can also be applied to the case of the hemisphere examined in $\S \mathrm{VI}$, so as to connect the potentials $V_{2}$ and $V_{0}$.

I have briefly indicated the necessary calculations below ; but it does not appear obvious that the transformation involved in the passage from (29) to (30) would necessarily convert the potential $V_{0}$ into the potential $V_{1}$, although it is evident that the integral (30) gives a solution of 
Laplace's equation, and it is also evident that with a more highly convergent integrand the two integrals (29) and (30) would be equal.

It is easy to modify the integrals given below so as to represent potentials corresponding to densities of the types mentioned by Mr. Watson; thus, if $\sigma=k s^{m}\{\log (a / s)\}^{l-1}$ in the disc, the integrals (29) and (30) will be multiplied by $\Gamma(l)$, and the denominators will be changed to $(m+2 t+2)^{l}$ and $(m+1-2 v)^{l}$ respectively. A corresponding modification can be made in (32) and (33), when the density of the hemisphere is $\rho=k s^{n-1}\{\log (a / s)\}^{l-1}$.

We begin by considering the potential of the disc defined in Section I. Taking the series (1) we can write

$$
\frac{(-1)^{n} C_{n}}{m+2 n+2}=\frac{(-1)^{n} \Gamma\left(n+\frac{1}{2}\right)}{\Gamma\left(\frac{1}{2}\right) \Gamma(n+1)(m+2 n+2)}
$$

and this is the residue at $t=n$ of the function

$$
-\frac{\Gamma(-t) \Gamma\left(t+\frac{1}{2}\right)}{\Gamma\left(\frac{1}{2}\right)(m+2 t+2)}
$$

Thus the series (1) can be expressed in the form*

$$
\left.V_{0}=\frac{2 \pi k a^{m+1}}{\Gamma\left(\frac{1}{2}\right)} \nmid \frac{1}{2 \pi \iota} \int \frac{\Gamma(-t) \Gamma\left(t+\frac{1}{2}\right)}{m+2 t+2} Z_{-(2 l+1)} d t\right\},
$$

where the integral is taken round the path marked $(0)$ in the diagram.
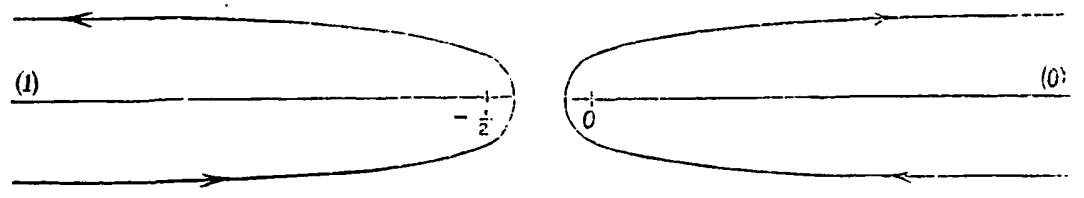

The integral (29) is convergent so long as $r>a$; but when $r<a$ the convergence fails. To convert the integral into a convergent form the path can be changed into (1); the value of the integral then gives

* We use here the notation for zonal harmonics

$$
Z_{u}=\left(r_{i} / a\right)^{n} P_{n}(|\cos \theta|),
$$

so that

$$
Z_{-(n+1)}=(a / v)^{n+1} P_{n}(|\cos 9|) ;
$$

because in general $P_{n}(\cos \theta)$ is defined by the hypergeometric series $F\left[-n, n+1,1, \frac{1}{2}(1-\cos \theta)\right]$, so that $P_{n}(\cos \theta)=P_{-(n+1)}(\cos \theta)$. 
the series (4) for $V_{1}$. To verify the truth of this statement write $t=-\left(\frac{1}{2}+v\right)$; then the path (1) of the $t$-plane is changed into the path (0) of the $v$-plane (allowing for the change of sign implied by the equation $d t=-d v$ ). Thus we find the integral

$$
\frac{2 \pi k a^{n+1}}{\Gamma\left(\frac{1}{2}\right)}\left\{\frac{1}{2 \pi \iota} \int \frac{\Gamma\left(v+\frac{1}{2}\right) \Gamma(-v)}{m+1-2 v} Z_{2 v} d v\right\},
$$

and when $m$ is not an odd integer the integral (30) at once yields the series

$$
\begin{aligned}
\frac{2 \pi k a^{m+1}}{\Gamma\left(\frac{1}{2}\right)}\left\{\sum_{0}^{\infty} \frac{(-1)^{n} \Gamma\left(n+\frac{1}{2}\right)}{(m+1-2 n) \Gamma(n+1)} Z_{2 n}\right. & \\
& \left.+\frac{1}{2} Z_{m+1} \Gamma\left(\frac{m}{2}+1\right) \Gamma\left(-\frac{m+1}{2}\right)\right\} .
\end{aligned}
$$

The series (31) can be identified with (4) by noting that

$$
\Gamma\left(\frac{m}{2}+1\right) \Gamma\left(-\frac{m+1}{2}\right)=-\frac{\pi \Gamma\left(\frac{1}{2} m+1\right)}{\cos \left(\frac{1}{2} m \pi\right) \Gamma\left(\frac{1}{2} m+\frac{3}{2}\right)}=+\frac{2 \sqrt{ } \pi}{p},
$$

where $p$ is the constant defined on p. 104 above.

When $m$ is an odd integer the integral (30) leads to the series (6); but the work involved hardly differs from that already given on pp. 104, 105.

In regard to the hemisphere of $\S \mathrm{VI}$, the present method will not serve to obtain $V_{1}$ from $V_{0}$, because $V_{1}$ does not satisfy Laplace's equation, whereas any integral such as (29) or (30) does satisfy it. On the other hand, it is not difficult to obtain $V_{2}$ from $V_{0}$ by using a complex integral.

For this purpose it will be simplest to change the sign of $\mu$ in (21) and in (24), so that the region now considered is that for which $\mu$ is positive; but the hemisphere is on the negative side of the $\operatorname{plan} \theta z=0$. We can then write (21) in the form

$$
\begin{gathered}
V_{0}=2 \pi k a^{n+1}\left\{\frac{1}{m+2} Z_{-1}+\sum_{0}^{\infty}(-1)^{n+1} \frac{K_{n}}{2 n+m+3} Z_{-(2 n+2)}\right\} \\
K_{n}=\frac{\Gamma\left(n+\frac{1}{2}\right)}{2 \Gamma\left(\frac{1}{2}\right) \Gamma(n+2)} .
\end{gathered}
$$

where

The last series leads at once to the integral

$$
V_{0}=\frac{2 \pi k a^{n+1}}{2 \Gamma\left(\frac{1}{2}\right)}\left\{\frac{1}{2 \pi \iota} \int \frac{\Gamma(t) \Gamma\left(-t-\frac{1}{2}\right)}{2 t+m+2} Z_{-2 t-1} d t\right\},
$$

taken round the path $(0)$ in the $t$-plane. 
Applying the same transformations as before, we find, when $r<a$, the integral

$$
\frac{2 \pi k a^{m+1}}{2 \Gamma\left(\frac{1}{2}\right)}\left\{\frac{1}{2 \pi \iota} \int \frac{\Gamma(v) \Gamma\left(-v-\frac{1}{2}\right)}{m+1-2 v} Z_{2 v} d v\right\},
$$

taken round the path $(0)$ in the $v$-plane; and this integral leads to the formula

(34) $2 \pi k a^{m+1}\left\{\frac{Z_{0}}{m+1}+\sum_{0}^{\infty} \frac{(-1)^{n} K_{n} Z_{2 n+1}}{2 n-m}+\frac{\Gamma\left(\frac{1}{2} m+\frac{1}{2}\right) \Gamma\left(-\frac{1}{2} m-1\right)}{4 \Gamma\left(\frac{1}{2}\right)} Z_{m+1}\right\}$.

By using the formula for $(m+1)(m+2) P_{m+1}(0)$ given on p. 120 , the series (34) can be identified with the series (24) for $V_{2}$, when the sign of $\mu$ is changed in the latter.

Similarly we cen derive the series corresponding to (27) from (33); but the work is essentially the same as that already given on pp. 120, 121.

To obtain the series for $V_{1}$ (in either case) it seems to be only possible to use the fact that the potential of the complete sphere at internal points is equal to

$$
\int_{0}^{r} k s^{m-1} \frac{4 \pi s^{2} d s}{r}+\int_{.}^{\prime \prime} k s^{m-1} \cdot 4 \pi s d s=\frac{4 \pi k}{m+1}\left(a^{m+1}-\frac{r^{m+1}}{m+2}\right),
$$

and so by subtracting from this expression the potential (34), we can infer a formula for $V_{1}$ which reduces to (23) or (26), according to the form of $m$. 